\title{
Smart quality control station for non-contact measurement of cylindrical parts based on confocal chromatic sensor
}

\author{
P. Chiariotti ${ }^{1 *}$, M. Fitti ${ }^{1}$, P. Castellini ${ }^{1}$, S. Zitti ${ }^{2}$, M. Zannini ${ }^{2}$, N. Paone ${ }^{1}$ \\ ${ }^{1}$ Dipartimento di Ingegneria Industriale e Scienze Matematiche (DIISM), Università \\ Politecnica delle Marche, Ancona, Italy \\ ${ }^{2}$ Zannini S.p.a., Castlfidardo, Italy \\ * corresponding author: p.chiariotti@univpm.it
}

\section{Introduction}

In modern digital factories, in-line quality control on $100 \%$ of production has become a key strategy to keep production processes and products under control. The real time and early identification of deviations and trends, performed at single process level, allow to prevent generation of defects and their propagation to down-stream processes, enabling the entire manufacturing system to perform early detection of process deviations and self-adaptation to different conditions. This is done with the purpose to approach the Zero-Defect target aimed by the manufacturing sector.

These concepts are the basis of recent European projects, among which GRACE [1] and GO0D MAN [2] represent significant examples. Both projects aim to integrate process and quality control in manufacturing systems [3][4]. GO0DMAN, in particular, focuses on multistage manufacturing production lines, and implements a distributed system architecture built on agent-based Cyber-Physical Systems (CPS) and smart in-line quality control systems. Smart quality control stations are designed to exhibit real-time adaptive behaviors, in order to keep measurement uncertainty under control even in case of variations of process/product parameters; they could implement data pre-processing to derive synthetic quality indicators, self-diagnosis and self-calibration to maximize the confidence level of the output.

The GO0D MAN project is based on a distributed system architecture typical of a multi-stage manufacturing system; Fig 1 shows the different functions that the hardware and software components are performing, as well as the flows of data and information that take place within the system. This architecture identifies a bottom level, the operational technology (OT) level, 
and an upper level, the Information Technology (IT) level. The architecture involves Cyber Physical Systems (CPS) which interact together. CPSs are the key enabling technology to reach the Zero-Defect target aimed by the manufacturing sector, thanks to its inherent distributed nature which allows to process data at local level and to share information between processes, in feed-back and feed-forward configurations. This enables early detection of defects and identification and correction of trends and anomalies.

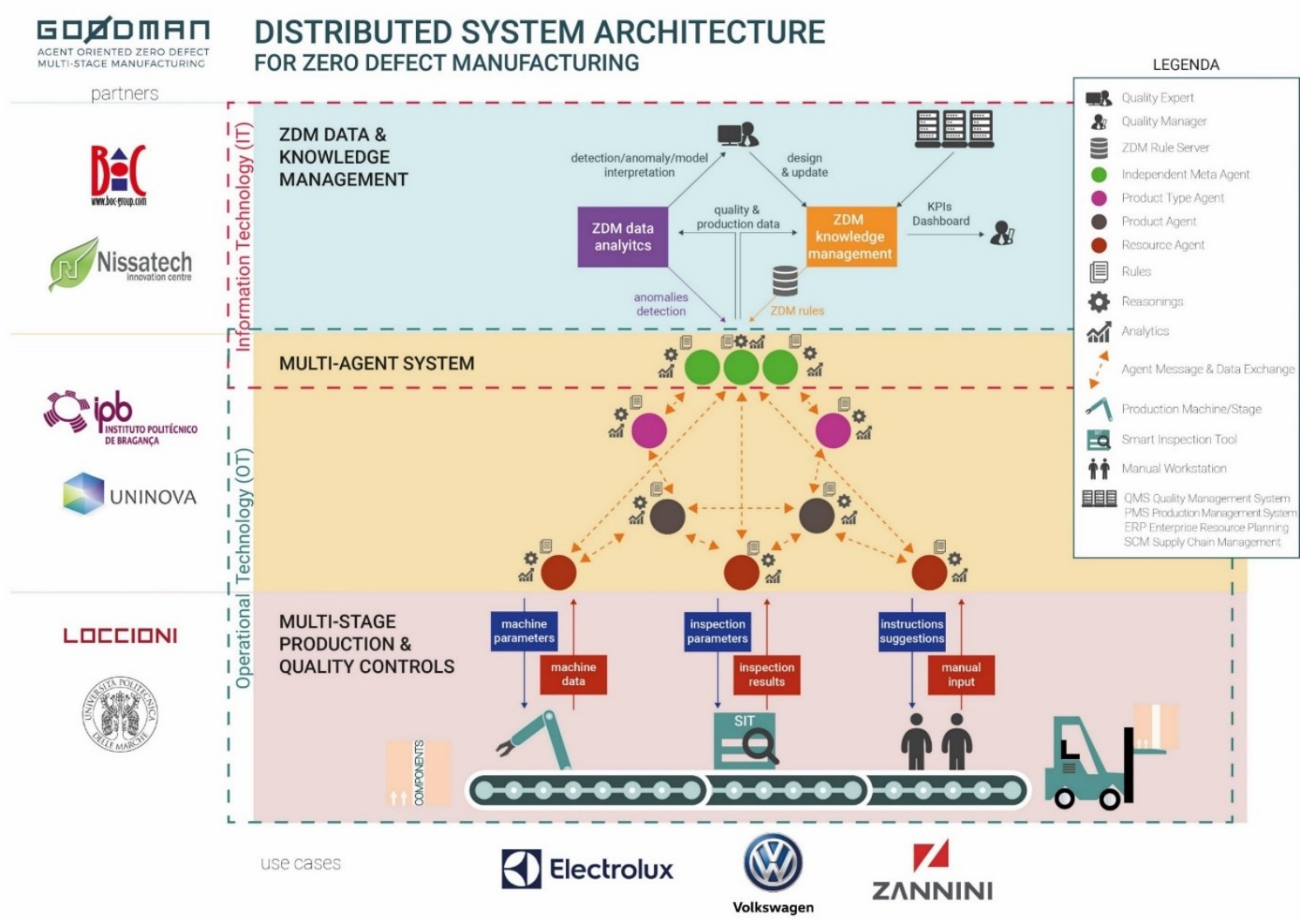

Fig. 1. GO0D MAN architecture (courtesy of GO0D MAN consortium [2]

In-line Quality Control Systems (QCS) perform diagnosis by measuring product parameters and comparing them to set specifications. QCSs that are located at the bottom layer of the system architecture; are by all means CPS. They are the nodes of the system where data are measured and quantitative information takes origin, and where diagnosis on process performance is done. The following data analytics and knowledge management relies on the quality of the information, hence on the metrological performance of the sensors used in QCSs.

Quality Control Systems are complex systems for testing and diagnosis. They are in charge of performing the test, taking the measurements, process data, perform diagnosis and provide 
output. They interact with the system through communication interfaces. QCSs can implement smart and adaptive behaviours, provided that the physical hardware of the QCS allows. Such behaviours are aimed to perform the required measurements while managing measurement uncertainty; therefore, QCS are a particular class of CPS.

Optical sensors are of relevant interest for in-line quality control, because they allow noncontact measurements, thus minimizing system intrusivity and maximizing speed of measurement.

Quality control is a decision making process, for which uncertainty of measurement plays a fundamental role [5][6]. The issue of measurement uncertainty and sensor calibration is therefore discussed in literature, to improve level of confidence of quality controls based on measurements; an example about dimensional measurements is discussed in [7].

Concerning manufacturing of high precision components, dimensional measurements are required for conformity assessment. High accuracy data can be acquired by Coordinate Measurement Machines (CMM)[8], which may employ contact or non-contact probes. However, CMMs are hardly usable in-line for $100 \%$ quality control due to long inspection times. The use of optical sensors for dimensional measurements opens the possibility of $100 \%$ in line quality control [9].

Among the different optical technologies which achieve micrometric resolution, chromatic confocal sensors (CCS) are good candidates for measuring internal diameter of cylindrical components. They are produced in small format and can measure in radial direction, down to the micrometric range. However, their metrological characteristics are affected by thermal disturbances which should be taken into account and compensated [9][10][11][12]. Chromatic confocal sensors measure distance at a point, which is the focal point of the sensor front lens; therefore, they are single point local sensors. In order to reconstruct the shape of the circular section of a turned part, the sensor has to be inserted inside the cylindrical part on-axis and then accurately scanned over the part under inspection; accuracy in scanning as well as accurate alignment is fundamental for the final result. Furthermore, their use as absolute distance sensors requires specific calibration and determination of the start of their measurement range.

This paper presents an automated quality control station based on a chromatic confocal sensors (CCS) which is rotated and scanned inside a cylinder, in order to measure its internal 
diameter. The station is a QCS which implements smart behaviors, in particular self-centering of the confocal probe and temperature compensation.

\section{The QCS test station}

The QCS test station (Fig. 2) is made of three main hardware components:

1. Chromatic confocal sensor for dimensional measurements;

2. Micrometric stages for automated motion;

3. Additional temperature sensor for ambient temperature assessment.

\section{The chromatic confocal sensors}

The CCS has a $90^{\circ}$ beam exit, so to make radial measurements inside small cavities and bores possible. The sensor holds the metrological characteristics reported in TABLE I.

TABLE I. Metrological characteristics of chromatic confocal sensor

\begin{tabular}{|c|l|}
\hline Measuring range & $6.5 \mathrm{~mm}$ \\
\hline Linearity & $13 \mu \mathrm{m} \leq \pm 0.2 \%$ Full Scale Output \\
\hline Resolution & $0.25 \mu \mathrm{m}$ \\
\hline Start Measuring Range (approx.) & $3.5 \mathrm{~mm}$ \\
\hline
\end{tabular}

The measurement distance measured by the sensor is intended from the sensor axis. However, the sensor itself is characterized by a "dead" measuring range inside which it is not possible to collect any useful data. Even though a precise knowledge of this value, identifying the so called "Start Measuring Range" (SMR), is not necessary in differential measurements, i.e. when relative distances between two different points are considered, it becomes important to fully characterize the sensor for absolute measurements as those described in this paper.

\section{The micrometric scanning system}

The micrometric stages allow for 4 independent degrees of freedom (DOF). The cylindrical sample under inspection is gripped by an electrical gripping chuck mounted on a 2 DOF X-Y traversing stage, while the confocal sensor is mounted on a 2 DOF scanning system, which make it possible:

1. insertion of the sensor into the part under inspection and vertical scanning along the $\mathrm{Z}$ axis; 
2. rotation of the sensor around its $\mathrm{Z}$ axis so to perform a circular scan of an inner section of the part.

3. Correction of the position of the part to compensate any eccentricity of the sensor

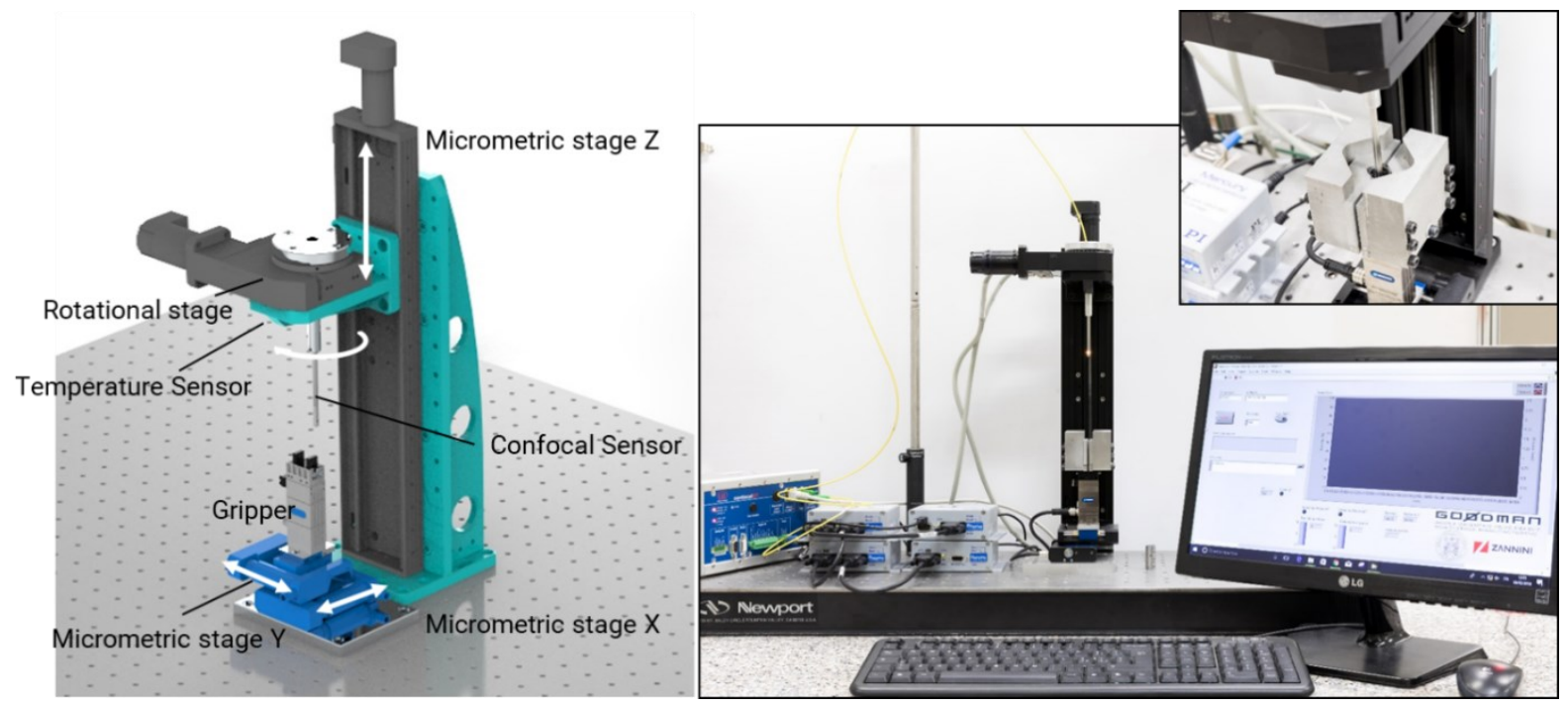

Fig. 2. Configuration of traversing stages

The main characteristics of the stages used in the QCS are reported in TABLE II.

TABLE II. Stages specification

\begin{tabular}{|c|c|c|}
\hline Stage & Travel range & Unidirectional repeatability \\
\hline X, Y linear stages & $25 \mathrm{~mm}$ & $0.1 \mu \mathrm{m}$ \\
\hline Rotational stage & $>360^{\circ}$ & $175 \mu \mathrm{rad}$ \\
\hline Z linear stage & $300 \mathrm{~mm}$ & $0.8 \mu \mathrm{m}$ \\
\hline
\end{tabular}

Indeed, to perform inner diameter measurements, a relative sensor-to-sample rotation is required. Rotating the sample around the sensor does not allow to correct an eventual eccentricity of the sensor with respect to the sample. Conversely, by rotating the sensor around its own axis, developing a procedure to take eccentricity into account a correction becomes possible.

\section{Smart behaviours for uncertainty management}

\section{Self-centering}

A correct alignment of the sensor axis with respect to the part longitudinal axis is of extreme importance to ensure an accurate measurement of part inner diameter during the sensor 
revolution. If eccentricity is present, the part diameter is assessed wrongly. To ensure a centering as much correct as possible the system performs a preliminary distance evaluation, at the same vertical position, on four points located every $90^{\circ}$ along the same circumference. An optimization procedure where the part is moved by the XY traversing axes, which means changing the relative position of the part longitudinal axis and the sensor vertical axis, runs to make these distances equal within a certain tolerance. The centering procedure is terminated when the stop criterion is reached. After this preliminary eccentricity correction, a circle fitting is carried out and finally an analysis of residuals with respect to the circle, so to refine the centering of the sensor with respect to the part.

\section{Temperature Self-compensation}

Ambient temperature represents a modifying effect for the chromatic confocal sensor it is necessary to compensate the erroneous distance value provided by the sensor. Indeed, temperature variations causes a twofold effect on the CCS:

- Onset of spurious displacement $\Delta z$

$$
\begin{aligned}
& n\left(\lambda_{0}, T_{0}\right)=n\left(\lambda_{0}+\Delta \lambda, T_{0}+\Delta T\right) \\
& \Delta \lambda=-\Delta \mathrm{T} \cdot\left(\frac{\partial \mathrm{n}}{\partial \mathrm{T}} / \frac{\partial \mathrm{n}}{\partial \lambda}\right) \\
& \Delta z=-\Delta \lambda\left(\frac{z_{\max }-z_{\min }}{B}\right)
\end{aligned}
$$

Where $n$ is the refraction index of the optics, $\lambda_{0}$ is the dominant wavelength recorded for an object at distance $z$ at the standard temperature $T_{0}$

- Variation of sensor working range $\left|d z_{\max }-d z_{\min }\right|$

$$
d z_{\min }=\frac{y^{2}}{\left(y-f_{\min }\right)^{2}}, d z_{\max }=\frac{y^{2}}{\left(y-f_{\max }\right)^{2}}
$$

where $f$ represents the effective focal length of the optics and $y$ stands for the distance (fixed) from the confocal fiber tip to the optics.

A natural consequence is that the working range increases with decreasing temperature and the sensor undergoes temperature drift. A dedicated measurement campaign was performed to experimentally characterize the correlation between ambient temperature and measured 
distance. The CCS was installed inside a temperature controlled chamber, together with a reference micrometer, arranged in a way to measure the distance to the moving plate of the micrometer (Fig. 3). Since it is expected that temperature variations cause different effects at different sensor-to-target distances $(z)$, the measurement campaign consisted in measuring five different sensor-to-target distances at different temperatures (Fig. 3). The nominal distances $\left(D_{n}\right)$ were evaluated by the micrometer, whose readout was also monitored by a custom-made visionbased system. The temperature range investigated ranged between $10^{\circ} \mathrm{C}$ and $40^{\circ} \mathrm{C}$.
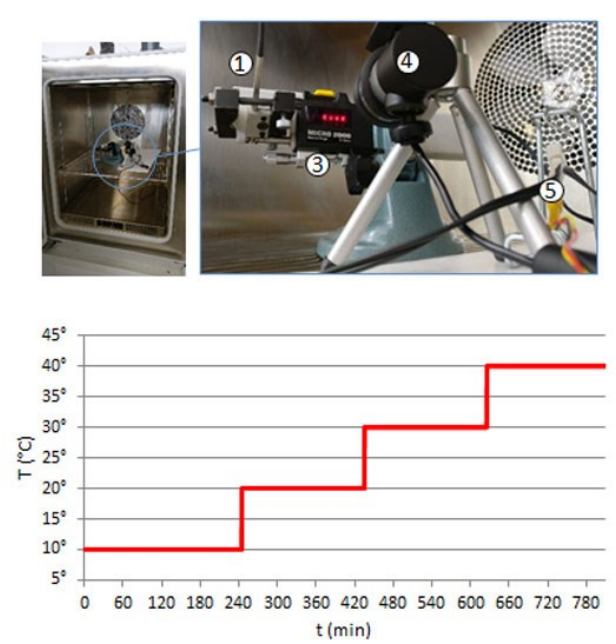

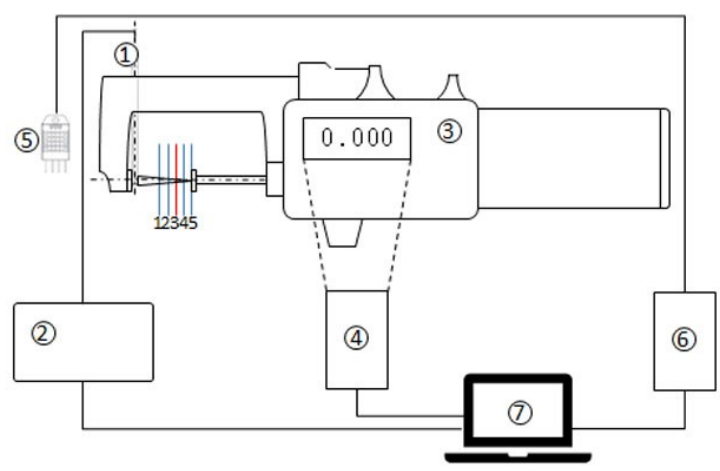

(1) Chromatic Confocal Sensor (CCS) (2) Chromatic Confocal Sensor Controller (5) Temperature sensor (3) Micrometer(res. $1 \mu \mathrm{m}$; accuracy $\pm 2 \mu \mathrm{m}$ ) (4) Webcam

Fig. 3. Test setup for temperature compensation: arrangement of the monitoring set-up (top-left), measuremnt chain (top-right) and temperature ranges investigated (bottom-left)

Data obtained made it possible to characterize the temperature drift of the sensor, which is approximately $8 \mu \mathrm{m} /{ }^{\circ} \mathrm{C}$. Moreover, by exploiting a superficial fitting of data collected during the tests, it becomes possible to get the sensor-to-target distance value $\left(D_{v}\right)$ given the distance value $(d)$ provided by the sensor and the ambient temperature $(T)$ measured close to the sensor. This allows temperature compensation.

\section{Self-calibration check}

It is important to periodically check the performance of the system so to ensure an uncertainty lower than $10 \mu \mathrm{m}$. This can be done by testing the correctness of both the temperature compensation and the self-centering approaches through the sequence of operations reported in the flow diagram of Fig. 4. In case any of the two steps fail, it becomes possible to perform 
eventual interventions on the system (e.g. check of the screwing torques of mechanical parts) so to keep performance at the desired levels.

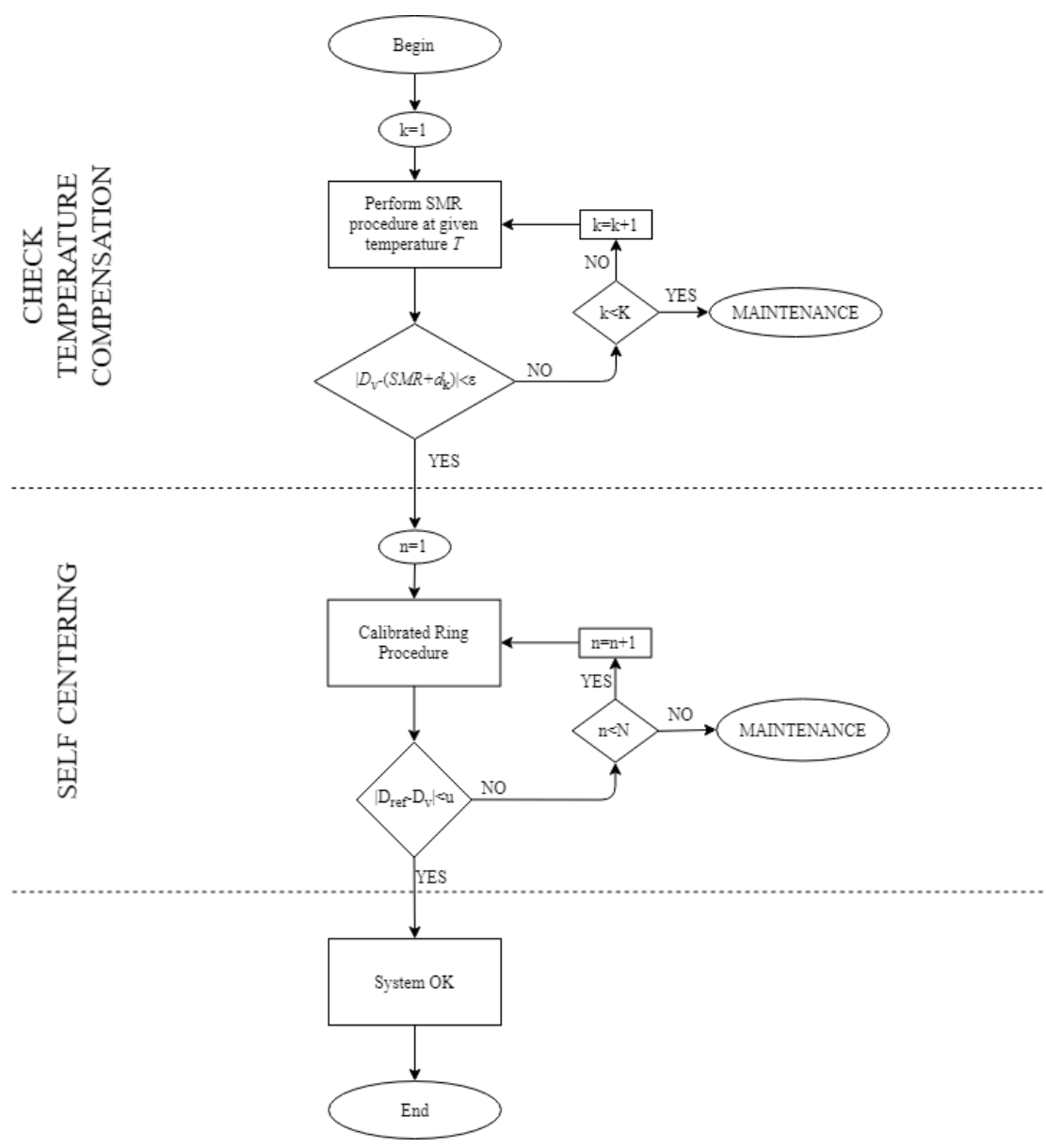

Fig. 4. Self-calibration check procedure

The SMR value at a known temperature can be calculated by running a dedicated two-steps procedure (Fig. 5) in which the sensor-to-target distance $\left(d_{i}\right)$ is evaluated on the opposite sides of a target of known thickness $(b)$ after moving the target by a known displacement value $(A)$ (e.g. by exploiting micrometric stages). A reference gauge block (thickness accurate up to micrometric values) can be exploited for the purpose. The SMR can then be extracted using Eq. (5)

$$
\mathrm{SMR}=\left(A-b-d_{1}-d_{2}\right) / 2 .
$$

The SMR value thus obtained is used to test the validity of the temperature compensation procedure. If data extracted by the distance-temperature fitting $\left(D_{v}\right)$ differs from the absolute 
value obtained as the summation of the SMR and the distance value provided by the sensor at $k^{\text {th }}$ step by more than a fixed threshold $\varepsilon$, the procedure has to be repeated until a maximum iteration number is reached. If the latter condition takes place, a maintenance operation is performed.
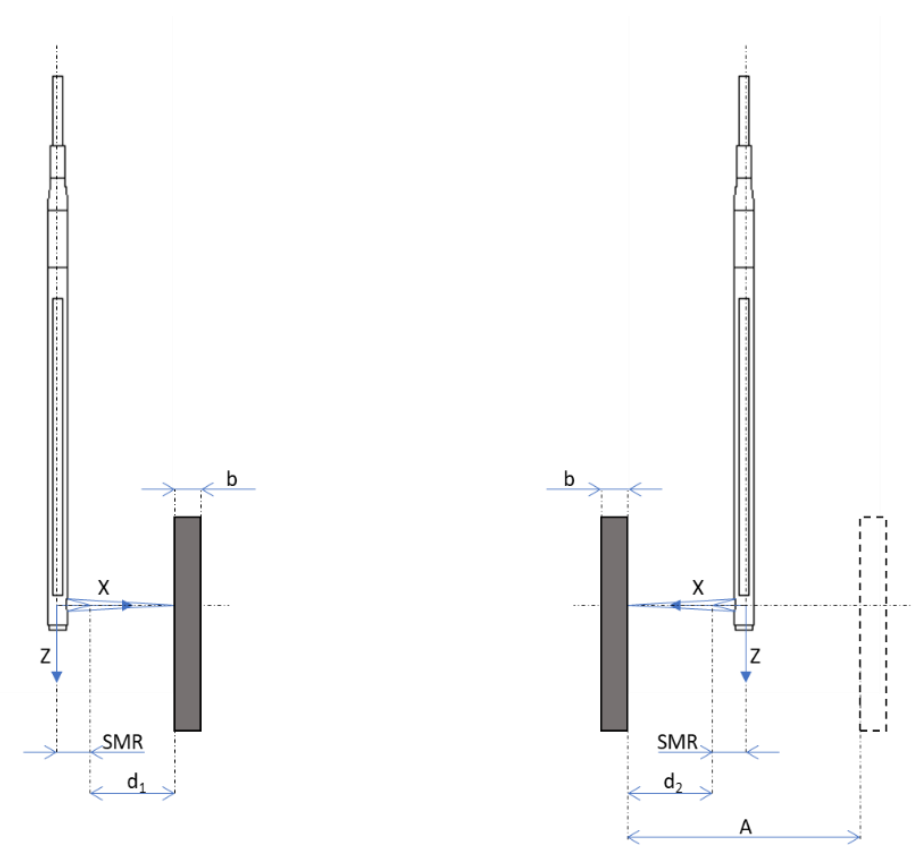

Fig. 5. SMR evaluation procedure: (i) sensor-to-target distance measured on the first side ot the reference target of known thickness (left); (ii) target moved by a known displacement value (A) and sensor-to-target distance measured on the opposite side of the target (right) with respect to (i)

In case the temperature compensation procedure succeeds, the efficacy of the self-centering approach is checked. To do so, a calibrated reference ring (micrometric accuracy) has to be used. The known radius value of the calibrated ring is compared to the radius estimated through the confocal sensor. If these two radii differ more than the target uncertainty, the self-centering procedure has to be repeated. This process iterates until either the former condition is verified or a maximum number of iteration $(N)$ is reached. If the self-centering verification process stops because the maximum number of iterations is reached, a maintenance operation has to be performed, otherwise the whole system is intended to be verified up to the desired uncertainty. 


\section{Results}

The QCS was tested on a sample of automotive sleeves to understand its capability in measuring the sleeve inner diameter and the location of the transversal holes along the sleeve height. For sake of shortness, only results from an internal circular scan of the sleeve, performed at a height of $2.4 \mathrm{~mm}$ from the sample bottom edge, are presented hereafter. On this section of the sleeve, a nominal diameter of $10.985 \mathrm{~mm}$ was expected. The whole circumference was scanned by a full rotation of the confocal sensor along its axis; radial measurements were taken at an angular sampling of $0.1^{\circ}$ at a rotation speed of $40^{\circ} / \mathrm{s}$.

The average diameter value on the section scanned results in $10.984 \mathrm{~mm}$ with a standard deviation of $0.009 \mathrm{~mm}$. The standard deviation was evaluated assuming data acquired over a whole rotation as constituting the statistical population. This value is compatible to the nominal expected diameter of the reference sample at that section height $(10.985 \mathrm{~mm})$, thus proving that the QCS, by exploiting its smart capabilities, i.e. self-centering and temperature selfcompensation, is able to provide highly accurate dimensional data. The residuals calculated with respect to the nominal radius are reported in Fig. 6 (b). A deviation, approximately in the order of $\pm 0.020 \mathrm{~mm}$, with respect to a circular shape is evident. 

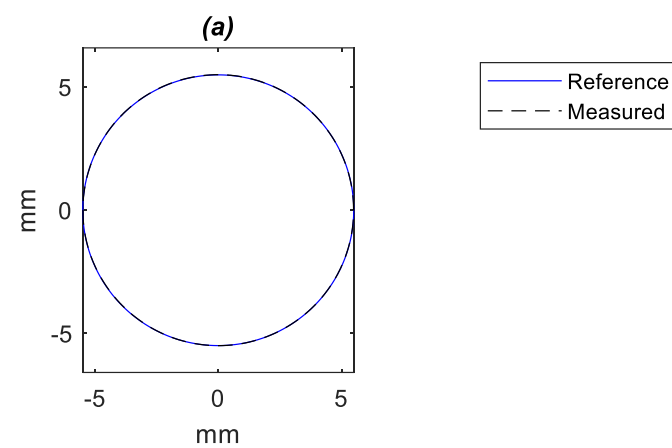

(b)

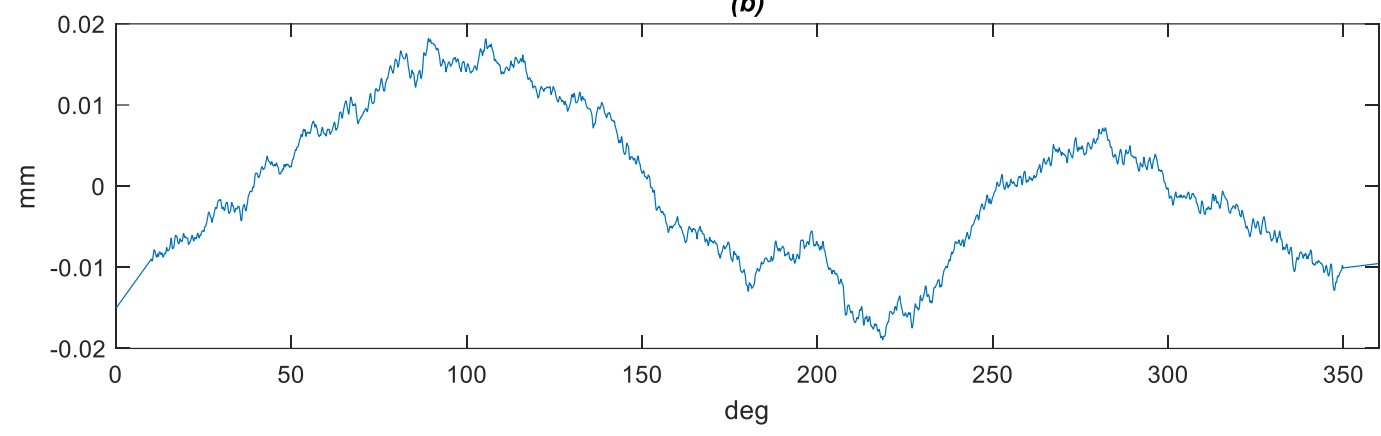

Fig. 6. Circular scan results: (a) nominal (blue solid line) vs measured (dashed black line) circumference of the sample (height of the scan: $2.4 \mathrm{~mm}$ from the bottom edge of the sample); (b) residuals of measured data with respect to nominal radius

\section{Conclusions}

A test station designed for high accuracy dimensional measurements of cylindrical parts has been presented. The station is intended to be employed for tests by the line. It is based on a chromatic confocal sensor, arranged with a $90^{\circ}$ beam exit, so to take radial measurements when inserted on-axis of a cylindrical part to be inspected. The sensor is mounted on a 4 degree of freedom traversing system, able to move it in $\mathrm{X}-\mathrm{Y}-\mathrm{Z}$ direction and rotate it axially, at micrometric accuracy.

Even if the sensor itself has remarkable metrological performance, in the $2 \mu \mathrm{m}$ range, its actual performance when mounted in the test station can be severely compromised by mounting tolerances, off-axis and eccentricity, as well as temperature disturbances, which can easily happen in an industrial environment. 
All these problems can be addressed and minimized if the test station is designed to perform smart behaviors. The paper has presented a self-centering procedure, which minimizes eccentricity, a self-compensation of temperature effects and a self- calibration check. Once implemented, such procedure allows to optimize metrological performance, reaching the target of an accuracy of $10 \mu \mathrm{m}$, which is a very tight requirement for an in-line measurement device.

\section{Acknowledgment}

This research is part of the European Project GO0DMAN-"aGent Oriented Zero Defect Multi-Stage Manufacturing". GO0D MAN project has received funding from the European Commission under the EU Framework Programme for Research and Innovation Horizon 2020 (2014 - 2020) within the FoF - Technologies for Factories of the Future initiative. Contract no. H2020-FOF-03-2016-723764.

\section{References}

[1] Web site of GRACE Project http://grace-project.org/ (accessed on March 13th, 2018)

[2] Web site of GO0DMAN Project: http://go0dman-project.eu/ (accessed on March 13th, 2018)

[3] P. Castellini, C. Cristalli, M. Foher, P.Leitao, N.Paone, I. Schjolberg, J. Tjønnås, C. Turrin, T. Wagner, Towards the integration of process and quality control using multi-agent technology, 37th Annual Conference of the IEEE Industrial Electronics Society, IECON 2011; Article number 6119347, Pages 421-426, Melbourne, VIC; Australia; November 2011

[4] C. Cristalli, M. Foehr, T. Jager, P.Leitao, N.Paone, P.Castellini, C. Turrin, I. Schjolberg, Integration of process and quality control using multi-agent technology, IEEE 22nd International Symposium on Industrial Electronics, ISIE 2013; Article number 6563737, Taipei; Taiwan; May 2013

[5] G.B. Rossi, F.Crenna, A probabilistic approach to measurement-based decisions, Measurement: Journal of the International Measurement Confederation, Volume 39, Issue 2, Pages 101-119, 2006 
[6] L. Mari ; D. Petri, The metrological culture in the context of big data: managing data-driven decision confidence, IEEE Instrumentation \& Measurement Magazine, vol. 20, issue 5, pp:4- 20, 2017

[7] G. D'Emilia, G. Di Rosso, A. Gaspari, A. Massimo, Metrological interpretation of a sixsigma process for improving the online optical measurement of automotive turbocharger dimensions, Proceedings of the Institution of Mechanical Engineers, Part D: Journal of Automobile Engineering, Volume 229, Issue 2, 13, Pages 261-269, 2015

[8] A. Weckenmann, T.Estler, G. Peggs, D. McMurtry, Probing systems in dimensional metrology, CIRP Annals - Manufacturing Technology, Volume 53, Issue 2, Pages 657-684, 2004

[9] G. Berkovic, E.Shafir, Optical methods for distance and displacement measurements, Advances in Optics and Photonics, Volume 4, Issue 4, Pages 441-471, 2012

[10] R. Hoenicka, A. Fink, Method for compensating for temperature realated measurement errors in a confocal chromatic measuring distance sensor, Patent No. US 8,248,598 B2, 2012.

[11] G. Berkovic, S. Zilberman, E. Shafir, Temperature Effects in Chromatic Confocal Distance Sensors, Proceedings of SENSORS, IEEE, Baltimore, MD, USA, 2013

[12] H. Nouira, N. El-Hayek, X. Yuan, N. Anwer, J. Salgado, Metrological characterization of optical confocal sensors measurements (20 and 350 travel ranges), $14^{\text {th }}$ Int. Conf. on Metrology and Properties of Engineering Surfaces, IOP Publishing, Journal of Physics: Conference Series 483, 012015, 2014

\section{Authors bio}

\section{$\underline{\text { Paolo Chiariotti }}$}

Paolo Chiariotti is researcher in Mechanical and Thermal Measurements at Università Politecnica delle Marche (Italy). He graduated in mechanical engineering in 2007 and received the $\mathrm{PhD}$ degree in Industrial Engineering and Management in 2011 from Università Politecnica delle Marche. His research interests are related to non-contact mechanical measurement techniques, mainly based on optical systems, signal and image processing and array acoustics. 


\section{$\underline{\text { Matteo Fitti }}$}

Matteo Fitti graduated in mechanical engineering at Università Politecnica delle Marche in 2016. He is currently enrolled in $\mathrm{PhD}$ program in Industrial Engineering at the same university. His research activity is focused on the development of innovative optical-based systems for quality control of turned components.

\section{$\underline{\text { Paolo Castellini }}$}

Paolo Castellini is associate professor at Università Politecnica delle Marche. Mechanical engineer graduated at Università of Ancona, he received the $\mathrm{PhD}$ degree in 1996 at University of Padova, Italy. He joined Università of Politecnica delle Marche as researcher in Mechanical and Thermal Measurements and Testing in 1997 and became Associate Professor in 2011. His research interests are related to non-contact mechanical measurement techniques, mainly based on laser and microphone arrays.

\section{$\underline{\text { Saverio Zitti }}$}

Saverio Zitti is Mechanical Engineer graduated at Università Politecnica delle Marche with Master in Technology and Innovation Management at BBS (Bologna Business School) and Master in Industry 4.0 Manufacturing Management at MIP (Politecnico di Milano's School of Management). He is business developer at Zannini S.p.a, with responsibility of several R\&D projects in cooperation with industrial and research partners.

\section{$\underline{\text { Marco Zannini }}$}

Marco Zannini is currently CEO of Zannini S.p.a. Founder of Zannini Poland Sp.zo.o in 2007 and responsible of Meccanica Veneta Srl acquisition in 2010, he has managed the international growth of the company into the manufacturing sector with main focus to the automotive supply chain of high precision metal components.

\section{$\underline{\text { Nicola Paone }}$}


Nicola Paone is full professor in Mechanical and Thermal Measurement at Università Politecnica delle Marche. Graduated in mechanical engineering in 1986, he was in 1986-87 researcher at Von Karman institute for Fluid Dynamics in Brussels and research engineer at FIAT Research Center in Turin in 1988. He then returned to academic career and became professor in 1992. His research is focused on the development and application of optical techniques to measure velocity, vibration and geometric quantities in several sectors. 